\title{
Análise da evidenciação ambiental nas corporações brasileiras de alto impacto ambiental listadas na BM\&FBOVESPA
}

\author{
Raiziane Cássia Freire da Silva \\ Mestre em Gestão Estratégica das Organizações pela Universidade Regional Integrada do Alto \\ Uruguai e das Missões (URI). E-mail: raifreire@ hotmail.com (Brasil)
}

\section{Rosane Maria Seibert}

Doutora em Ciências Contábeis pela Universidade do Vale do Rio dos Sinos (Unisinos). Professora na Universidade Regional Integrada do Alto Uruguai e das Missões, no curso de Mestrado Profissional em Gestão Estratégica das Organizações. E-mail: rseibert@ santoangelo.uri.br (Brasil)

\section{Lucélia Ivonete Juliani}

Doutora em Energia pela Universidade Federal do ABC (UFABC). Professora na Universidade Federal do Pampa (Unipampa). E-mail: lucelia.juliani@gmail.com (Brasil)

\section{Berenice Beatriz Rossner Wbatuba}

Doutora em Desenvolvimento Regional pela Universidade de Santa Cruz do Sul. Professora Universidade Regional Integrada do Alto Uruguai e das Missões, no curso de Mestrado Profissional em Gestão Estratégica das Organizações. E-mail: bwbatuba@santoangelo.uri.br (Brasil)

\section{RESUMO}

O objetivo deste estudo foi o de identificar quais são as informações ambientais que as corporações brasileiras, classificadas como de alto impacto ambiental listadas na BM\&FBOVESPA, divulgam em seus sítios de internet. A amostra foi constituída por 43 corporações. A abordagem metodológica utilizada foi quantitativa e a coleta de evidências foi bibliográfica, documental e observação não participante nos sites das corporações que compõem a amostra. Os dados foram tratados por meio de análise de conteúdo, estatística descritiva e análise de variância (Anova). Os resultados demonstram que a categoria "Outras informações ambientais" é a mais divulgada, enquanto "Políticas ambientais" é o indicador com maior nível de evidenciação. Também aponta o segmento de "Celulose e papel" como o que mais disponibiliza informações ambientais. Quando as informações são analisadas por segmento constata-se o baixo nível de evidenciação, o que indica a necessidade do aumento de divulgação de informações ambientais pelas corporações. A análise da variância indica que as médias não são iguais entre os indicadores expostos, o que confirma os diferentes níveis de evidenciação das corporações classificadas como de alto impacto ambiental.

Palavra-Chave: Evidenciação ambiental. Teoria da legitimidade. Corporações de alto impacto ambiental. 


\title{
Analysis of Environmental Disclosure in Brazilian High Environmental Impact Corporations Listed on BM\&FBOVESPA
}

\begin{abstract}
The objective of this study was to identify the environmental information that Brazilian corporations, classified as having high environmental impact listed on the BM\&FBOVESPA, disclose on their websites. The sample consisted of 43 corporations. The methodological approach used was quantitative and the collection of evidence was bibliographical, documentary and non-participant observation in the sites of the corporations that compose the sample. The data were treated by means of content analysis, descriptive statistics and analysis of variance (ANOVA). The results show that the category "Other environmental information" is the most widely publicized, while "Environmental policies" is the indicator with the highest level of disclosure. It also points to the segment of "pulp and paper" as the one that provides the most environmental information. When the information is analyzed by segment, the low level of disclosure is evident, which indicates the need for increased disclosure of environmental information by corporations. The analysis of variance indicates that the averages are not equal among the exposed indicators, which confirms the different levels of disclosure of corporations classified as having high environmental impact.
\end{abstract}

Keyword: Environmental disclosure. Legitimacy theory. High environmental impact corporations.

\section{Como referenciar em APA:}

Silva, R. C. F., Seibert, R. M., Juliani, L. I., Wbatuba, B. B. R. (2018). Análise da Evidenciação Ambiental nas Corporações Brasileiras de Alto Impacto Ambiental Listadas na BM\&FBOVESPA. R.G.Secr.,GESEC, 9(3).

\section{Como referenciar em ABNT:}

SILVA, R. C. F.; SEIBERT, R. M.; JULIANI, L. I.; WBATUBA, B. B. R. Análise da Evidenciação Ambiental nas Corporações Brasileiras de Alto Impacto Ambiental Listadas na BM\&FBOVESPA. R.G.Secr.,GESEC, v. 9, n. 3, Dez. 2018.
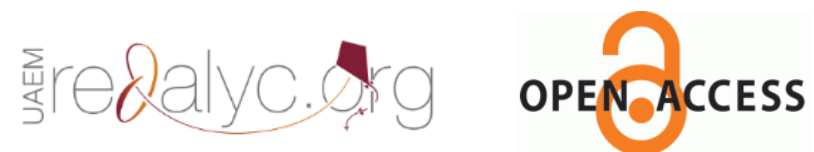


\section{Introdução}

Apesar de não serem recentes as discussões sobre as questões ambientais (Burgwal \& Vieira, 2014; Yusoff, Lehman \& Nasir, 2006), a conjuntura atual do meio ambiente tem intensificado os debates e as campanhas globais de conscientização para o uso sustentável dos recursos naturais e para a preservação ambiental (Nossa, 2002; Yusoff et al., 2006; Rover, Borba \& Murcia, 2009; Burgwal \& Vieira, 2014; Ortas, Gallego-Alvarez \& Etxerberria, 2014; Correa, Gonçalves \& Moraes, 2015;). Essa preocupação é estendida às corporações que também causam impacto no ambiente através de suas ações (Correa et al., 2015), e por isso, elas são chamadas a adotarem uma conduta ambientalmente responsável (Nossa, 2002; Yusoff et al., 2006; Ortas et al., 2014).

As corporações não desempenham um papel meramente econômico na sociedade, devendo os resultados gerados por suas atividades maximizar não apenas os interesses dos proprietários, mas também de todas as outras partes envolvidas (Freeman, 2002; O’Donovan, 2002; Yusoff et al., 2006; Parmar et al., 2010), dentre eles as questões associadas ao meio ambiente. É por meio da evidenciação que as corporações informam aos seus stakeholders os resultados obtidos por elas, ou seja, demonstram estar cumprindo com as expectativas sociais para obter legitimação (Shocker \& Sethi, 1973; Patten 1992; Lindblom, 1994; Suchman, 1995; Deegan, 2002; O’Donovan, 2002; Michelon, 2011; Cormier \& Magnan, 2015).

A evidenciação ambiental refere-se à divulgação de informações de caráter ambiental e não é regulamentada no Brasil (Nossa, 2002; Murcia et al., 2009; Rover, Tomazzia, Murcia \& Borba, 2012; Correa et al., 2015; Marquezan, Seibert, Bartz, Barbosa \& Alves, 2015), portanto sua adesão é voluntária. No entanto, a divulgação voluntária demonstra as estratégias ambientais consequentes de um compromisso (Michelon, 2011; Clarkson, Xiaoshua, Li \& Richardson, 2013) da corporação com seus stakeholders legitimadores (Freeman, 2002; Phillips, 2003; Parmar et al., 2010; Michelon, 2011), produzindo benefícios (Clarkson et al, 2013; Correa et al., 2015) como: alinhamento de valores corporação-sociedade; antecipação aos ataques de grupo de pressão; melhoria da reputação e imagem da corporação; e demonstração de responsabilidade socioambiental (O’Donovan, 2002; Michelon, 2011).

Em se tratando de corporações de alto impacto ambiental, a evidenciação de informações ambientais se faz particularmente relevante devido à pressão social sofrida em decorrência da condição agressiva de suas atividades ao meio ambiente (Nossa, 2002). Logo, 
os stakeholders esperam evidenciar informações que demonstrem o apoio dado, por essas corporações, as causas ambientais e os esforços para reduzir os impactos de suas atividades (Yusoff et al., 2006; Ortas et al., 2014; Correa et al., 2015). Em outras palavras, os stakeholders têm expectativas de que as corporações divulguem seus impactos ambientais e defendam suas ações protetivas e regeneradoras, o que demonstraria a vontade de manter ou recuperar a legitimidade por meio da evidenciação de informações que atendam os interesses dos stakeholders legitimadores (Elijido-Ten, Kloot \& Clarson, 2010). Ou seja, a evidenciação ambiental contribui para o fortalecimento ou recuperação da legitimidade, haja vista a divulgação dessas informações influenciar na percepção dos stakeholders quanto à legitimação da corporação (Cormier \& Magnan, 2015).

Por outro lado, a divulgação acarreta custos e a evidenciação voluntária tende a acontecer quando os benefícios são maiores que os custos (Watts \& Zimmerman, 1978; Verrecchia, 1983; 2001; Dye, 1986; Murcia \& Santos, 2009). Nesse sentido, a Internet surge como uma aliada para minimizar esses custos (Morhardt, 2010; Fifka, 2013), pois, em suas páginas eletrônicas, as corporações, principalmente aquelas que mais afetam o meio ambiente, podem divulgar relatórios de informações ambientais com custo baixo, como forma de evitar a pressão social por prestação de contas (Marquezan et al., 2015; O’Donovan, 2002; Patten, 2002). Assim, diante do contexto sobre a importância da evidenciação, em especial das informações ambientais, motivadas pela preocupação com cenário ambiental e a possibilidade de divulgação com custo baixo, esta pesquisa busca responder à seguinte questão: Quais são as informações ambientais evidenciadas nas páginas eletrônicas de Internet das corporações com grande potencial de impacto ao meio ambiente? Para obter a resposta, o estudo objetivou identificar quais são as informações ambientais evidenciadas nas páginas eletrônicas da Internet, pelas corporações caracterizadas como de alto impacto ambiental listadas na BM\&FBOVESPA.

Além desta introdução, este artigo apresenta a revisão da literatura que fundamentou este estudo, descreve o processo metodológico adotado para o desenvolvimento da pesquisa, os resultados encontrados e a discussão deles. Finalizando, têm-se as considerações finais.

\section{Revisão da Literatura}

Para fundamentar teoricamente a pesquisa desenvolvem-se, na sequência, dois temas: a evidenciação ambiental e a teoria da legitimidade, como motivadora dessa evidenciação. 
Desde a década de 1990, a evidenciação ambiental tem despertado o interesse corporativo e científico (Deegan, 2002; Nossa, 2002; Yusoff et al., 2006; Fifka, 2013; Burgwal \& Vieira, 2014; Correa et al., 2015) e devido a fatores como desmatamentos, aquecimento global, poluição, consumo dos recursos naturais, desastres ambientais provocados por grandes corporações as atenções dispensadas às informações relacionadas ao meio ambiente estão se ampliando (Nossa, 2002; Yusoff et al., 2006; Fifka, 2013; Burgwal \& Vieira, 2014; Ortas et al., 2014; Correa et al., 2015).

Entende-se por evidenciação a divulgação tempestiva de informações qualitativas e quantitativas detalhadas, relevantes e compreensíveis para que seus usuários possam entender os riscos e as atividades das corporações (Dantas, Zendersky \& Niyama, 2004). Logo, a evidenciação ambiental diz respeito à divulgação das informações referente aos eventos que afetam o meio ambiente, direta ou indiretamente, e refletem no resultado financeiro da corporação (Macêdo, Moura, Dagostini \& Hein, 2013; Correa et al., 2015; Marquezan et al., 2015).

A evidenciação pode ocorrer de forma compulsória ou voluntária, mas normalmente ocorre da primeira forma (Dantas et al., 2004; Beuren \& Sothe, 2009; Beuren, Hein \& Boff, 2011). A compulsória, refere-se às informações prestadas em decorrência de exigências legais dos órgãos reguladores e entidades de classe. As voluntárias são todas as informações prestadas sem a obrigatoriedade legal de evidenciação (Beuren \& Sothe, 2009; Beuren et al., 2011). No Brasil, a legislação não obriga que haja evidenciação ambiental por parte das corporações (Murcia et al., 2009; Rover et al., 2012; Macêdo et al., 2013; Correa et al., 2015; Marquezan et al., 2015), logo, a aderência é voluntária ficando a critério dessas corporações evidenciar tais informações, bem como o conteúdo a ser divulgado, ainda que sejam somente fatos e atos favoráveis à sua imagem (Deegan \& Rankin, 1996).

A evidenciação voluntária, inclusive das ações ambientais, é explicada por várias razões, dentre elas destacam-se as expostas na Tabela 1.

Tabela 1 - Explicações teóricas para evidenciação voluntária.

\begin{tabular}{l|l}
\hline \multicolumn{1}{c|}{ Motivação } & \multicolumn{1}{c}{ Autores } \\
\hline $\begin{array}{l}\text { Pressão pública e o desejo de evitar ou } \\
\text { minimizar a intervenção política em suas }\end{array}$ & $\begin{array}{l}\text { Patten, 1992; Deegan, 2002; O’Donovan, 2002; Yusoff et al., } \\
\text { atividades, por meio de restrições legais, } \\
\text { tributárias, dentre outras. }\end{array}$ \\
\hline
\end{tabular}


Análise da evidenciação ambiental nas corporações brasileiras de alto impacto ambiental listadas na BM\&FBOVESPA

Em função do desempenho econômico, já que a divulgação ambiental afeta direta e indiretamente o mercado econômico.

Intuito de atrair investidores socialmente responsáveis.

Para melhorar a reputação e transmitir uma imagem positiva da corporação.

A responsabilidade da corporação com seus stakeholders, reconhecendo-lhes o direito de participarem deu suas políticas e ações ambientais, principalmente quando estas causam danos externos.

Para cumprir a expectativa da comunidade como consequência do contrato social, previamente assumido.

Para identificar os determinantes da extensão da evidenciação ambiental.

Fonte: Elaborada pelas autoras.

Normalmente a evidenciação não acontece por uma única razão, mas por vários motivos que se inter-relacionam (Deegan, 2002) e impulsionam as corporações a tal feito. Todavia, essas motivações são respaldadas por algumas teorias sociais que evoluíram em busca de clarificar o comportamento social das corporações (O'Donovan, 2002; Yusoff et al., 2006), dentre elas a Teoria da Legitimidade, que é amplamente utilizada para justificar (Deegan, 2002; Bebbingont et al., 2008; Ortas et al., 2014; Cormier \& Magnan, 2015), e provavelmente é a que mais justifica (O’Donovan, 2002), a importância da evidenciação ambiental.

As corporações são criações sociais regidas por um contrato social firmado com a sociedade na qual elas estão inseridas. Nesse contrato são identificadas as expectativas explícitas (determinadas por lei) e as implícitas (aquelas que a lei não abrange) que devem ser cumpridas pelas partes (Shocker \& Sethi, 1973; Deegan, 2002; O’Donovan, 2002). Assim, a teoria da legitimidade parte da premissa de que uma corporação só é legítima quando cumpre com as expectativas explícitas e implícitas desse contrato social, devolvendo algo do interesse da sociedade para merecer permanecer nela (Shocker \& Sethi, 1973; Lindblom, 1994; Suchman, 1995).

A legitimidade é uma percepção generalizada de que as corporações estão agindo de forma desejável, apropriada ou adequada conforme as normas, valores, crenças e definições construídas dentro da sociedade à qual pertencem (Lindblom, 1994; Suchman, 1995). Esses
Yusoff et al., 2006; Mir \& Rahaman, 2011; Clarkson et al.,

Deegan, 2002; Yusoff et al., 2006; Murcia et al., 2009;

Patten, 1992, Deegan \& Rankin, 1996; Deegan, 2002;

O’Donovan, 2002; Bebbingont, Larrinaga \& Moneva, 2008;

Deegan \& Rankin, 1996; Freeman, 2002; Nossa, 2002; Clarson, 2010; Parmar et al., 2010; Michelon, 2011; Mir \& Rahaman, 2011.

Patten, 1992; Deegan \& Rankin, 1996; Deegan, 2002;

Murcia, et al. 2008; Marquezan et al., 2015; Rover, Murcia \& Murcia, 2015; Ezhilarasi \& Kabra, 2017. 
valores sociais são inconstantes e podem ameaçar a legitimidade da corporação quando esta não conseguir acompanhar tais mudanças e, uma vez perdida a legitimidade, o contrato social pode ser revogado provocando penalidades econômicas, sociais ou jurídicas (Lindblom, 1994; Deegan \& Rankin, 1996; Deegan, 2002; O’Donovan, 2002; Correa et al., 2015).

São os stakeholders que detêm o poder de legitimar as corporações (Freeman, 2002; Phillips, 2003; Parmar et al., 2010). Todavia, é necessário que as corporações evidenciem informações, a fim de demonstrarem aos seus stakeholders que as expectativas sociais e ambientais estão sendo cumpridas e a legitimidade possa ser reconhecida (Shocker \& Sethi, 1973; Dye, 1986; Patten 1992; Deegan, 2002; O’Donovan, 2002; Michelon, 2011; Cormier \& Magnan, 2015). O tipo de informação a ser evidenciada pela corporação está vinculado ao objetivo da legitimação (Correa et al., 2015), o que indica o valor estratégico da escolha da divulgação (Wagenhofer, 1990; Michelon, 2011; Cho et al., 2012). Em virtude disso, na maioria dos casos, são divulgadas informações positivas que promovem uma imagem favorável da corporação, omitindo-se as informações negativas para não prejudicar a legitimação (Patten, 1992; Deegan \& Rankin, 1996; Verrecchia, 2001; Deegan, 2002; Cho \& Patten, 2007; Comier \& Magnan, 2013).

Desse modo, destaca-se a relevância do papel desempenhado pela evidenciação como ferramenta de legitimação. Por meio dela, as corporações podem gerenciar a percepção dos stakeholders para conseguir ou manter sua posição de legítima (Patten, 1992; Lindblom, 1994; Deegan, 2002; O’Donovan, 2002; Cho \& Patten, 2007; Bebbingont et al., 2008; Comier \& Magnan, 2013, Correa et al., 2015). Por essa razão, as corporações de alto impacto ambiental adotariam a evidenciação ambiental como meio de enfrentar as possíveis ameaças à sua legitimidade, por serem mais expostas e suscetíveis à pressão pública (Patten, 1992; Cho \& Patten, 2007; Michelon, 2011; Rover et al., 2012; Comier \& Magnan, 2013; Burgwal \& Vieira, 2014). Afinal, quanto maior for a percepção desfavorável da atuação da corporação na sociedade, maior será o seu interesse em mudá-la para manter ou recuperar a legitimidade organizacional (O’Donovan, 2002).

É por meio da evidenciação que as corporações demonstram para seus stakeholders que seus objetivos, produtos e atividades estão de acordo com as expectativas implícitas e explícitas do contrato social, inclusive aquelas relacionadas ao meio ambiente, para que a legitimidade possa ser reconhecida. E, na iminência de perda da legitimidade, também é por 
meio da evidenciação que as corporações podem mudar sua situação, pois do contrário os esforços para legitimação seriam considerados insuficientes (Patten, 1992; Lindblom, 1994; Deegan 2002; Freeman, 2002; O’Donovan, 2002; Phillips, 2003; Cho \& Patten, 2007; Bebbingont et al., 2008; Parmar et al., 2010; Michelon, 2011; Cho et al., 2012; Cormier \& Magnan, 2015; Correa et al., 2015).

A evidenciação pode ser realizada por meio de indicadores, que são uma medida representativa de questões ou preocupações específicas (Rempel et al., 2004), neste caso, referente a assuntos ambientais. Os indicadores ambientais são caracterizados pela sua capacidade de quantificar, mensurar, simplificar e comunicar informações relevantes para análise e tomada de decisão (Gallopin, 1996). Assim, eles agregam e facilitam a comunicação de informações complexas e abstratas tornando-as mais compreensíveis, práticas e quantificáveis, quando necessário (Hammond et al., 1995; Hardi \& Barg, 1997; Van Bellen, 2004).

Os indicadores ambientais podem estar contidos em relatórios como, de sustentabilidade, de responsabilidade social e balanços sociais, que são os mais adotados pelas corporações devido à ausência de um modelo padrão para divulgação dessas informações (Nossa, 2002; Murcia et al., 2009; Michelon, 2011; Rover et al., 2012; Fifka, 2013; Marquezan et al., 2015). Por outro lado, também podem estar contidos em outro meio de comunicação; por exemplo, as páginas eletrônicas da Internet que vêm sendo utilizadas como ferramentas de evidenciação proporcionando um alcance maior de públicos, com baixo custo (Morhardt, 2010; Fifka, 2013), visto que as corporações devem ser transparentes e prestadoras de contas buscando um equilíbrio entre os custos e os benefícios de evidenciar (Verrecchia, 1983; 2001; Dye, 1986).

A seguir, apresentam-se os procedimentos metodológicos utilizados para a pesquisa.

\section{Procedimentos Metodológicos}

Este estudo é uma pesquisa de cunho quantitativo e descritivo (Vergara, 2010), cuja coleta de evidências foi realizada, primeiramente, por meio de pesquisa bibliográfica para revisão da literatura. Em seguida, ocorreu a pesquisa documental e observação não participante nas páginas eletrônicas de Internet das corporações pertencentes à amostra. Com base na Lei n. ${ }^{\circ} 10.165$, de 27 de dezembro de 2000, identificaram-se os segmentos classificados como de alto impacto ambiental, sendo: Extração e Tratamento de Minerais; 
Indústria Química; Indústria Metalúrgica; Indústria de Celulose e Papel; Transporte, Terminais e Depósito de Comércio, e Indústria de Couro e Pele. Este último segmento não possui corporações listadas na Bolsa de Valores, Mercadorias e Futuro de São Paulo (BM\&FBOVESPA).

O universo desta pesquisa foi de 75 corporações listadas na BM\&FBOVESPA, em 18 de maio de 2016. A amostra resulta em 43 corporações, pois no caso de corporações pertencentes ao mesmo grupo empresarial, com website em comum, foi selecionada apenas uma por grupo. Na Tabela 2 demonstra-se a composição da amostra por segmento:

Tabela 2 - Demonstrativo do universo e amostra da pesquisa por segmento.

\begin{tabular}{l|c|c}
\multicolumn{1}{c|}{ Segmento } & Universo & Amostra \\
\hline Extração e Tratamento de Minerais - ETM & 14 & 8 \\
\hline Indústria Química - IQ & 35 & 14 \\
\hline Indústria Metalúrgica - IM & 12 & 10 \\
\hline Indústria de Celulose e Papel - ICP & 7 & 6 \\
\hline Transporte, Terminais e Depósito de Comércio - TTDC & 7 & 5 \\
\hline \multicolumn{1}{c|}{ Total } & 75 & 43 \\
\hline
\end{tabular}

Fonte: Dados da pesquisa.

Para a coleta de dados, inicialmente, definiram-se os indicadores ambientais a partir da revisão da literatura teórica e empírica de pesquisas publicadas por Patten (1992); Nossa (2002); Yusoff et al. (2006); Murcia et al. (2009); Rover et al. (2009); Rover et al. (2012); Santos Silva \& Macagnan (2012); Macêdo et al. (2013); Souza, Sousa, Parente, Ferreira \& Souza Filho (2013); Burgwal \& Vieira (2014); Marquezan et al. (2015); Correa et al. (2015); bem como a NBC T 15 (Conselho Federal de Contabilidade, 2004) e documentos de orientação de evidenciação, tal como o Global Reporting Iniciate (GRI, 2015) e Indicadores de Responsabilidade Social do Instituto Ethos de Empresas e Responsabilidade Social (Instituto Ethos, 2013). A relação desses indicadores está demonstrada na Tabela 3:

Tabela 3 - Categorias e indicadores de evidenciação ambiental.

\begin{tabular}{l|ll}
\hline \multicolumn{1}{c|}{ Categorias Ambientais } & \multicolumn{1}{c}{ Indicadores Ambientais } \\
\hline \multirow{2}{*}{$\begin{array}{l}\text { Políticas, projetos e parcerias } \\
\text { ambientais }\end{array}$} & 1. & Políticas ambientais \\
\cline { 2 - 3 } & 2. & Projetos ambientais \\
\hline
\end{tabular}


Análise da evidenciação ambiental nas corporações brasileiras de alto impacto ambiental listadas na BM\&FBOVESPA

\begin{tabular}{|c|c|}
\hline & 3. Parcerias ambientais \\
\hline & 4. Prêmio por proteção ambiental \\
\hline & 5. Participação em índices de sustentabilidade \\
\hline \multirow{2}{*}{ Sistema de gerenciamento ambiental } & 6. Certificações ambientais (ISO 14000, 14001, outras) \\
\hline & 7. Auditoria ambiental \\
\hline \multirow{5}{*}{$\begin{array}{l}\text { Impacto dos produtos e processos no } \\
\text { meio ambiente }\end{array}$} & 8. Desperdícios / Gerenciamento de resíduos \\
\hline & 9. Reciclagem \\
\hline & 10. Odor \\
\hline & 11. Vazamentos e derramamentos \\
\hline & 12. Reparo de danos ambientais \\
\hline \multirow{3}{*}{ Energia e água } & 13. Conservação e/ou uso eficiente de energia \\
\hline & 14. Uso eficiente e/ou reutilização da água \\
\hline & 15. Tratamento de efluentes \\
\hline \multirow{4}{*}{ Informações financeiras ambientais } & 16. Investimentos ambientais \\
\hline & 17. Gastos ambientais \\
\hline & 18. Passivo / Provisões ambientais \\
\hline & 19. Seguro ambiental \\
\hline \multirow{2}{*}{ Educação, treinamento e pesquisa } & 20. Educação ambiental (interno e/ou comunidade) \\
\hline & 21. Pesquisas relacionadas ao meio ambiente \\
\hline \multirow{5}{*}{ Outras informações ambientais } & 22. Emissão de gases efeito estufa \\
\hline & 23. Reflorestamento \\
\hline & 24. Preservação / Conservação da biodiversidade \\
\hline & 25. Controle de poluição \\
\hline & 26. Envolvimento dos Stakeholders relacionado ao meio ambiente \\
\hline
\end{tabular}

Fonte: Dados da pesquisa.

Ressalta-se que o indicador reflorestamento, a princípio, pode não se aplicar para alguns segmentos, entretanto acredita-se que toda e qualquer corporação pode reflorestar em consonância com a Lei ambiental $n^{\circ}$. 12.651/2012.

Após a definição dos indicadores representativos de informações ambientais, usou-se a opção de busca dos sites das corporações para identificar a presença ou não desses indicadores. Não obtendo resultado por meio dessa ferramenta, procedeu-se a leitura de relatórios e documentos disponíveis para consulta, bem como se procedeu observação não participante em todo o site com o mesmo objetivo. Por meio de uma planilha Excel foram relacionadas todas as corporações da amostra e todos os indicadores identificados na revisão 
da literatura. Para a informação evidenciada, atribuiu-se peso um e para a informação não evidenciada, peso zero. Depois, foi feita a soma dos indicadores evidenciados e calculado o percentual de evidenciação por segmento, por corporação, por categoria e por indicadores, configurando-se como os índices de evidenciação ambiental.

Para interpretar se a informação evidenciada equivaleria ao indicador proposto, utilizou-se a análise de conteúdo que, segundo Bardin (1977), é um conjunto de técnicas de análise de comunicações que, por intermédio de procedimentos sistemáticos e objetivos de descrição do conteúdo, possibilita a inferência de conhecimentos relativos às variáveis inferidas das mensagens. Os resultados dessa análise são os índices de evidenciação ambiental. As evidências também foram tratadas por meio de estatística descritiva, como média e desvio padrão para identificar a existência de padrões de evidenciação, e análise de variância das médias (Anova) como forma de validação dos resultados.

\section{Apresentação dos Resultados}

A partir dos indicadores identificados nos sítios de internet das corporações brasileiras listadas na BM\&FBOVESPA, procedeu-se a divisão dos resultados em três partes: por segmentos, categorias e indicadores. Na Tabela 4 é possível verificar o nível de evidenciação ambiental por segmentos:

Tabela 4 - Média de indicadores ambientais evidenciados por segmentos.

\begin{tabular}{l|c|c|c|c}
\hline \multicolumn{1}{c|}{ Segmentos } & $\begin{array}{c}\mathbf{N}^{\mathbf{0}} \\
\text { Corporações }\end{array}$ & $\begin{array}{c}\text { Média de } \\
\text { Indicadores } \\
\text { Evidenciados }\end{array}$ & $\begin{array}{c}\% \\
\text { Desvio } \\
\text { Padrão }\end{array}$ \\
\hline Indústria de Celulose e Papel - ICP & 6 & 17,50 & 67,31 & 5,75 \\
\hline Extração e Tratamento de Minerais - ETM & 8 & 10,88 & 41,83 & 9,08 \\
\hline Indústria Química - IQ & 14 & 9,00 & 34,62 & 8,04 \\
\hline Indústria Metalúrgica - IM & 10 & 8,90 & 34,23 & 8,39 \\
\hline Transporte, Terminais e Depósito de Comércio - TTDC & 5 & 8,80 & 33,85 & 9,04 \\
\hline
\end{tabular}

Fonte: Dados da pesquisa.

Observando os resultados apresentados na Tabela 4, constata-se que as corporações pertencentes ao segmento de Indústria de Celulose e Papel foi o que mais evidenciou 
indicadores ambientais em suas páginas eletrônicas da Internet. Encontrou-se um total de 17,50 indicadores evidenciados, em média, que corresponde a 67,31\% dos 26 indicadores esperados. O segmento de Extração e Tratamento de Minerais aparece em segundo lugar em evidenciação de informações ambientais apresentando, em média, 41,83\% dos 26 indicadores.

As corporações do segmento de Transporte, Terminais e Depósitos de Comércio evidenciaram uma média de 8,80 indicadores, demonstrando ser o setor com o menor índice de evidenciação. Destaca-se que, quanto maior é a média de evidenciação, menor é o desvio padrão e vice-versa, o que indica menor e maior variância na evidenciação, respectivamente. Ou seja, quando a média de evidenciação é alta, há baixa variação nos indicadores evidenciados e quando a média de evidenciação é baixa, há uma variância maior nos indicadores evidenciados por cada uma das corporações.

O segmento de Papel e Celulose também apresenta os maiores níveis de evidenciação em quase todas as categorias, com exceção da categoria informações financeiras ambientais, como pode ser observado na Tabela 5. Essa tabela apresenta a média e o desvio padrão por segmento e por categoria de evidenciação:

Tabela 5 - Média de indicadores ambientais por categorias evidenciados versus segmentos.

\begin{tabular}{|c|c|c|c|c|c|c|c|c|c|c|c|c|c|}
\hline \multirow{2}{*}{ Categorias } & \multirow{2}{*}{$\begin{array}{l}\mathbf{N}^{\circ} \\
\text { Ind. }\end{array}$} & \multicolumn{10}{|c|}{$\begin{array}{c}\text { Média de indicadores por categorias } \\
\text { evidenciados por segmentos }\end{array}$} & \multicolumn{2}{|c|}{ Geral } \\
\hline & & $\operatorname{ICP}^{\mathrm{a}}$ & $\mathbf{D} \mathbf{P}^{\mathrm{b}}$ & $\operatorname{ETM}^{c}$ & $\mathbf{D P}$ & $\mathbf{I Q}^{\mathrm{d}}$ & DP & $\mathbf{I M}^{\mathrm{e}}$ & DP & $\begin{array}{c}\text { TT } \\
\text { DC }^{\mathrm{f}}\end{array}$ & DP & Méd. & DP \\
\hline $\begin{array}{l}\text { Políticas, projetos } \\
\text { e parcerias } \\
\text { ambientais }\end{array}$ & 5 & 3,50 & 1,64 & 1,75 & 1,67 & 2,14 & 1,70 & 1,40 & 1,26 & 2,00 & 1,87 & 2,07 & 1,67 \\
\hline $\begin{array}{ll}\text { Sistema } & \text { de } \\
\text { gerenciamento } & \\
\text { ambiental } & \end{array}$ & 2 & 1,67 & 0,82 & 1,00 & 1,07 & 0,64 & 0,93 & 1,20 & 1,03 & 0,80 & 1,10 & 1,00 & 1,00 \\
\hline $\begin{array}{lr}\text { Impacto } & \text { dos } \\
\text { produtos } & \mathrm{e} \\
\text { processos } & \text { no } \\
\text { ambiente } & \end{array}$ & 5 & 3,00 & 1,10 & 1,88 & 1,96 & 1,57 & 1,40 & 1,10 & 1,37 & 1,40 & 1,52 & 1,70 & 1,54 \\
\hline Energia e água & 3 & 2,33 & 0,82 & 1,13 & 1,36 & 1,57 & 1,34 & 1,30 & 1,42 & 1,00 & 1,41 & 1,47 & 1,32 \\
\hline $\begin{array}{l}\text { Informações } \\
\text { financeiras } \\
\text { ambientais }\end{array}$ & 4 & 1,00 & 1,26 & 1,50 & 1,60 & 0,71 & 1,07 & 0,80 & 0,92 & 0,60 & 0,89 & 0,91 & 1,15 \\
\hline $\begin{array}{l}\text { Educação, } \\
\text { treinamento } \\
\text { pesquisas }\end{array}$ & 2 & 1,33 & 0,82 & 1,00 & 0,93 & 0,36 & 0,63 & 0,80 & 0,92 & 1,00 & 1,00 & 0,79 & 0,86 \\
\hline $\begin{array}{l}\text { Outras } \\
\text { informações } \\
\text { ambientais }\end{array}$ & 5 & 4,67 & 0,82 & 2,63 & 1,92 & 2,00 & 2,08 & 2,30 & 2,26 & 2,00 & 2,00 & 2,56 & 2,07 \\
\hline
\end{tabular}


Fonte: Dados da pesquisa.

${ }^{\mathrm{a}}$ ICP - Industria de Celulose e Papel; ${ }^{\mathrm{b}} \mathrm{DP}$ - Desvio Padrão; ${ }^{\mathrm{c}}$ ETM - Extração e Tratamento de Minerai; ${ }^{\mathrm{d}}$ IQ Industria Química; ${ }^{\mathrm{e}} \mathrm{IM}$ - Industria Metalúrgica; ${ }^{\mathrm{f}} \mathrm{TTDC}$ - Transporte, Terminais e Deposito de Comércio.

Nota-se que o segmento de Papel e Celulose se destaca com as maiores médias de indicadores evidenciados nas categorias políticas, projetos e parcerias ambientais, sistema de gerenciamento ambiental, impacto dos produtos e processos no meio ambiente, energia e água, educação, treinamento e pesquisas e outras informações ambientais. O segmento de Extração e Tratamento de Minerais foi o setor que mais evidenciou indicadores da categoria informações financeiras ambientais, mas apresenta um desvio padrão de 1,60 demonstrando haver alta variação na evidenciação dos indicadores pelas corporações deste segmento.

O segmento da Indústria Metalúrgica destaca-se com as menores médias de evidenciação nas categorias políticas, projetos e parcerias ambientais e impacto dos produtos e processos no meio ambiente, além de apresentar altos desvios padrão demonstrando a variação nos indicadores evidenciados. O segmento de Transporte, Terminais e Depósito apresentou a menor média de evidenciação na categoria de informações financeiras ambientais, com desvio padrão de 0,89 , e na categoria energia e água, com desvio padrão 1,41, confirmando a variação nos indicadores evidenciados dentro de cada categoria.

A Indústria Química destaca-se com a menor média de evidenciação na categoria sistemas de gerenciamento com desvio padrão de 0,93 , e na categoria educação, treinamento e pesquisas com desvio padrão de 0,63 , também apresentando elevada variação nos indicadores evidenciados. A categoria outras informações ambientais apresentou 2,0 como média tanto no segmento da Indústria Química como no segmento de Transportes, Terminais e Depósito de Comércio, com desvio padrão de 2,08 e 2,00, respectivamente. Desse modo, confirma-se que as menores médias de evidenciação apresentam os maiores desvios padrão, o que indica alta variação entre os indicadores evidenciados pelas corporações de cada segmento.

A categoria outras informações ambientais, na análise global, destaca-se por possuir a maior média de corporações evidenciado os cinco indicadores, conforme demonstrado na Tabela 6.

Tabela 6 - Média de indicadores ambientais evidenciados por categoria versus corporações.

\begin{tabular}{c|c|c|c|c}
\hline Ordem & Categorias & $\begin{array}{c}\text { Média de indicadores } \\
\text { evidenciados }\end{array}$ & $\%$ & $\begin{array}{c}\text { Desvio } \\
\text { padrão }\end{array}$ \\
\hline
\end{tabular}


Análise da evidenciação ambiental nas corporações brasileiras de alto impacto ambiental listadas na BM\&FBOVESPA

\begin{tabular}{c|l|c|c|c}
\hline 1 & Outras informações ambientais & 22,00 & 51,16 & 5,87 \\
\hline 2 & Sistema de gerenciamento ambiental & 21,50 & 50,00 & 0,71 \\
\hline 3 & Energia e água & 21,00 & 48,84 & 1,00 \\
\hline 4 & Políticas, projetos e parcerias ambientais & 17,80 & 41,40 & 10,08 \\
\hline 5 & Educação, treinamento e pesquisas & 17,00 & 39,53 & 4,24 \\
\hline 6 & Impacto dos produtos e processos no meio ambiente & 14,60 & 33,95 & 9,40 \\
\hline 7 & Informações financeiras ambientais & 9,75 & 22,67 & 5,74 \\
\hline
\end{tabular}

Fonte: Dados da pesquisa.

É possível verificar que a categoria outras informações ambientais teve 51,16\% dos seus indicadores evidenciados. Ou seja, das 43 corporações, em média 22 evidenciaram todos os indicadores, porém houve variação na evidenciação dos indicadores, uma vez que o desvio padrão foi de 5,87. A segunda categoria mais evidenciada, sistema de gerenciamento ambiental, aparece próxima à primeira com $50 \%$ dos seus indicadores divulgados, porém é a categoria com menor variação na evidenciação dos indicadores pelas corporações, conforme demonstra o desvio padrão de 0,71 .

O menor número de indicadores evidenciados ocorreu na categoria informações financeiras ambientais, apresentado apenas $22,7 \%$ de evidenciação, com desvio padrão de 5,74. Apesar de os indicadores da categoria políticas, projetos e parcerias ambientais apresentarem um nível de 41,40\% de evidenciação dos seus indicadores pelas corporações, foi a categoria que mais apresentou variação com um desvio padrão de 10,08, ressaltando a oscilação entre os indicadores evidenciados pelas corporações.

Analisando por indicadores, observa-se que políticas ambientais é o mais evidenciado pelas corporações, conforme demonstrado na Tabela 7:

Tabela 7 - Total de evidenciação dos indicadores ambientais.

\begin{tabular}{l|c|c}
\hline \multicolumn{1}{c|}{ Indicadores } & $\begin{array}{c}\text { Total } \\
\text { Evidenciados }\end{array}$ & $\%$ \\
\hline Políticas ambientais & 33 & 76,7 \\
\hline Controle de poluição & 27 & 62,8 \\
\hline Preservação / Conservação da biodiversidade & 25 & 58,1 \\
\hline Desperdícios / Gerenciamento de resíduos & 24 & 55,8 \\
\hline Envolvimento dos stakeholders relacionado ao meio ambiente & 24 & 55,8 \\
\hline Reciclagem & 23 & 53,5 \\
\hline Certificações ambientais (ISO 14000, 14001, outras) & 22 & 51,2 \\
\hline
\end{tabular}




\begin{tabular}{|c|c|c|}
\hline Emissão de gases efeito estufa & 22 & 51,2 \\
\hline Projetos ambientais & 22 & 51,2 \\
\hline Tratamento de efluentes & 22 & 51,2 \\
\hline Auditoria ambiental & 21 & 48,8 \\
\hline Conservação e/ou uso eficiente de energia & 21 & 48,8 \\
\hline Educação ambiental (interno e/ou comunidade) & 20 & 46,5 \\
\hline Uso eficiente e/ou reutilização da água & 20 & 46,5 \\
\hline Investimentos ambientais & 17 & 39,5 \\
\hline Reparo de danos ambientais & 16 & 37,2 \\
\hline Prêmio por proteção ambiental & 15 & 34,9 \\
\hline Pesquisas relacionadas ao meio ambiente & 14 & 32,6 \\
\hline Parcerias ambientais & 12 & 27,9 \\
\hline Reflorestamento & 12 & 27,9 \\
\hline Passivo / Provisões ambientais & 10 & 23,3 \\
\hline Gastos ambientais & 9 & 20,9 \\
\hline Participação em índices de sustentabilidade & 7 & 16,3 \\
\hline Vazamentos e derramamentos & 7 & 16,3 \\
\hline Odor & 3 & 7,0 \\
\hline Seguro ambiental & 3 & 7,0 \\
\hline
\end{tabular}

Fonte: Dados da pesquisa.

Também obtiveram altos índices os indicadores controle de poluição e preservação/conservação da biodiversidade, com $62,8 \%$ e $58,1 \%$ de evidenciação, respectivamente. $\mathrm{O}$ indicador envolvimento dos stakeholders, relacionado ao meio ambiente, teve um índice razoável de evidenciação $(55,8 \%)$ pelas corporações. Apesar de o indicador participação em índices de sustentabilidade ter obtido 16,3\% de divulgação, as corporações que expuseram esse indicador apresentaram índice de evidenciação relevante (Braskem S.A e Fribria Celulose S.A com 88,46\%; Natura Cosméticos S.A com 84,62\%; Kablin S. A com 80,77\%; Vale S. A com 76,92\%; Suzano Papel e Celulosa S. A com 69,23\% e a Gerdau S. A $\operatorname{com} 53,85 \%)$.

\section{Análise de confiabilidade e validade dos resultados}

Para confirmar os resultados encontrados previamente, utilizou-se o software Grett 1.9.10 (2015) para realizar a análise de variância (Anova), que se trata de um método 
estatístico pelo do qual se procede o teste de igualdade de médias (Martins e Domingues, 2011, p. 378). Para tanto, propôs-se as seguintes hipóteses: $\mathrm{H}_{0}$ - Pelo menos duas médias de tratamento são iguais e $\mathrm{H}_{1}$ - As médias de tratamento não são iguais. O resultado é apresentado na Tabela 8, a seguir.

Tabela 8 - Resultados da Anova.

\begin{tabular}{l|c|c|c|c|c}
\cline { 2 - 5 } & Soma dos quadrados & Gl & Quadrado médio & F & Sig. \\
\hline Entre grupos & 34,183 & 25 & 1,367 & 6,357 &, 000 \\
Nos grupos & 234,884 & 1092 &, 215 & & \\
Total & 269,067 & 1117 & & \\
\hline
\end{tabular}

Fonte: Dados da pesquisa.

O teste $\mathrm{F}$ calculado é de 6,357 maior que o F crítico 1,52 (6,357>1,52). Assim, com $1 \%$ de significância, rejeita-se a hipótese nula de que pelo menos duas médias são iguais. Como o p-valor foi de 0,000 , não se rejeita a hipótese 1: as médias da evidenciação dos indicadores representativos de informações ambientais não são iguais, confirmando as médias e os desvios padrões apresentados anteriormente. Isso indica que as corporações não seguem um padrão de divulgação e tem estratégias de evidenciação ambiental diferentes, mesmo que tenham o mesmo objetivo, a busca pela legitimidade organizacional.

Os resultados apresentados neste tópico são discutidos na sequência.

\section{Discussão dos Resultados}

Os dados demonstrados na Tabela 1 confirmam resultados anteriores, como de Rover et al. (2009) e Souza et al (2013), onde o segmento de Papel e Celulose apresenta maior nível de evidenciação. Mesmo com o espaço de tempo transcorrido entre essas pesquisas é possível perceber que esse segmento ainda se mantém como o que mais evidencia informações aos seus stakeholders. Isto pode ser justificado pelo fato desse setor ser considerado um dos que mais degrada o meio ambiente (apesar de todos serem considerados de alto impacto ambiental) e, por isso, tem a necessidade de assegurar sua legitimidade e evitar pressões públicas devido ao seu tipo de atividade econômica (Patten, 1992; Deegan, 2002; O'Donovan, 2002; Yusoff et al. 2006; Cho \& Patten, 2007; Beuren \& Sothe, 2009; Michelon, 2011; Cho et 
al., 2012; Rover et al., 2012; Cormier \& Magnan, 2015; Fifka, 2013; Souza et al., 2013; Ortas et al., 2014).

Esse mesmo argumento pode ser usado para explicar o motivo pelo qual o segmento de Extração e Tratamento de Minerais aparece em segundo lugar em evidenciação de informações ambientais. Também é um dos setores considerados como mais degradante e poluente do meio ambiente e quanto mais sensíveis ambientalmente, maior tende a ser o nível de evidenciação ambiental das corporações (Cho \& Patten, 2007; Michelon, 2011; Burgwal \& Vieira, 2014; Cormier \& Magnan, 2015). Dentre as corporações pertencentes a esse segmento, a Petrobras apresentou o maior número de indicadores ambientais, evidenciando 23 dos 26 indicadores pesquisados, corroborando com Borba \& Murcia (2009) e Murcia et al. (2009).

De modo geral, quando se comparam os cincos segmentos, constata-se que o nível de evidenciação ambiental se mostrou baixo, haja vista somente um setor ter obtido acima de $50 \%$ de indicadores evidenciados, quando a teoria preconiza que a evidenciação de informações ambientais é uma ferramenta estratégica de legitimação, principalmente para corporações de alto impacto ambiental por estarem mais expostas e serem mais suscetíveis à pressão dos stakeholders e às regulamentações ambientais (Patten, 1992; Deegan, 2002; Yusoff et al., 2006; Cho \& Patten, 2007; Michelon, 2011; Ortas et al., 2014). Essa informação corrobora com os estudos de Macêdo et al. (2013) e Souza et al. (2013), ao alertar sobre a necessidade do aumento do nível de evidenciação ambiental das corporações, especialmente, quando se tratam de corporações classificadas como de alto impacto ambiental.

Os resultados demonstrados na Tabela 2, em que o segmento de Papel e Celulose apresenta os maiores níveis de evidenciação por categoria, ratifica a pesquisa de Rover et al. (2009) ao apontá-lo como o que mais evidenciou informações ambientais em quase todas as categorias. $\mathrm{O}$ indicador sistema de gerenciamento ambiental ter-se apresentado como a segunda categoria mais evidenciada, conforme demonstrado na Tabela 3, corrobora com pesquisas anteriores como de Yusoff et al. (2006) e Macêdo et al. (2013). Para Yusoff et al. (2006), o sistema de gestão ambiental é um sinalizador para os stakeholders de que a corporação está buscando melhorar suas ações ambientais; por isso é considerado um fator relevante que afeta a legitimidade (Cormier \& Magnan, 2015). 
Ainda na Tabela 3, o resultado que demonstra a categoria informações financeira ambientais como a que teve o menor número de indicadores evidenciados, ratifica os resultados encontrados por Rover et al. (2009) e Macêdo et al. (2013) e chamam atenção para pouca transparência de informações que servem para tomada de decisão de potenciais investidores, aumento do valor da corporação (Clarkson et al., 2013), melhoria da imagem empresarial e aceitação dos stakeholders responsáveis pela legitimação da corporação (Patten, 1992; Lindblom, 1994; Deegan \& Rankin, 1996; Verrecchia, 2001; Deegan 2002; O’Donovan, 2002; Freeman, 2002; Phillips, 2003; Cho \& Patten, 2007; Murcia \& Santos, 2009; Parmar et al., 2010; Michelon, 2011; Cho et al., 2012; Macêdo et al., 2013; Cormier \& Magnan, 2015; Correa et al., 2015; Marquezan et al., 2015).

A análise por indicadores, demonstrada na Tabela 4, constata que políticas ambientais é o mais evidenciado pelas corporações. Tal resultado corrobora com os trabalhos de Yusoff et al. (2006); Murcia et al. (2009); Rover et al. (2009); Macêdo et al. (2013); Souza et al. (2013); Correa et al. (2015). Ao evidenciar suas políticas ambientais, as corporações demonstram crer na reponsabilidade com o meio ambiente e estar comprometidas com ele, além de antever informações que respondam possíveis questionamentos dos stakeholders acerca do assunto (Yusoff et al., 2006).

O acreditar no papel de zelar pelo meio ambiente também pode justificar a segunda e terceira posição dos indicadores controle de poluição e preservação/conservação da biodiversidade, respectivamente. Além disso, esses indicadores proporcionam informações positivas, sustentando a teoria de que normalmente as corporações tendem a divulgar mais informações que sejam favoráveis a sua imagem e lhes garantam a legitimação por parte dos stakeholders (Patten, 1992; Deegan \& Rankin, 1996; Verrechia, 2001; Deegan, 2002; Nossa, 2002; O’Donovan, 2002; Murcia \& Santos, 2009; Michelon, 2011; Ortas et al., 2014).

O indicador envolvimento dos stakeholders relacionado ao meio ambiente, que demonstrou um índice razoável de evidenciação, demonstra que as corporações estão preocupadas em envolver seus stakeholders nas políticas organizacionais. O bom desempenho da corporação está ligado com a boa relação com seus stakeholders, que devem ser reconhecidos e considerados tanto na estrutura e política organizacional da corporação quanto na tomada de decisões, pois o equilíbrio dessa relação é fundamental para sua legitimação e sua continuidade (Freeman, 2000; O’Donovan, 2002; Phillips, 2003; Parmar et al., 2010).

Os estudo de Murcia et al.(2009), Rover et al.(2012) e Marquezan et al.(2015), demonstrando que corporações presentes em índices de sustentabilidade tendem a evidenciar 
mais informações ambientais do que as demais, são confirmados pela presente pesquisa, visto que, as corporações que divulgaram esse indicador apresentaram um índice de evidenciação relevante.

É importante ressaltar que a ausência de evidenciação dos indicadores ambientais usados na pesquisa, não denota a inexistência dessas informações nas corporações, uma vez que foram analisadas somente aquelas divulgadas em suas páginas eletrônicas de Internet. Porém, os stakeholders buscam pela evidenciação ambiental e as corporações têm o dever de comunicar informações que sejam de interesse deles (Deegan \& Rankin, 1996; Freeman, 2002; Nossa, 2002; O’Donovan, 2002; Phillips, 2003; Yusoff et al., 2006; Parmar et al., 2010) e as páginas eletrônicas de Internet são ferramentas de comunicação acessíveis, entre as corporações e seus stakeholders, que podem ser usadas para esse fim (Morhardt, 2010; Fifka, 2013). Ademais, a falta de evidenciação prejudicaria a permanência das corporações na comunidade onde estão inseridas, pois pode ocasionar a perda da credibilidade dos stakeholders e, consequentemente, a perda da legitimidade (Patten, 1992; Suchman, 1995; Lindblom, 1994; Deegan, 2002; O’Donovan, 2002; Phillips, 2003).

\section{Considerações Finais}

O presente estudo teve por objetivo identificar os tipos de informações ambientais que as corporações brasileiras, classificadas como de alto impacto ambiental e listadas na BM\&FBOVESPA, evidenciam em suas páginas eletrônicas da Internet. Para tanto, foram investigadas um total de 43 corporações pertencentes aos segmentos: Extração e Tratamento de Minerais, Indústria Química, Indústria Metalúrgica, Indústria de Celulose e Papel e Transporte, Terminais e Depósito de Comércio.

Foi realizada uma análise segmental onde observou-se que a Indústria de Celulose e Papel foi a que mais evidenciou informações ambientais em suas páginas eletrônicas de Internet, com 67,31\% das informações esperadas e um desvio padrão de 5,75. Esse resultado corrobora com alguns estudos anteriores como o de Rover et al., 2009 e o de Souza et al., 2013. O segmento que apresentou o menor nível de evidenciação ambiental foi o de Transporte, Terminais e Depósitos Comerciais, com 33,85\% dos indicadores possíveis e um desvio padrão de 9,04 . 
Por categoria, o segmento de Celulose e Papel também aparece com o maior índice de evidenciação, em quase todas as categorias, confirmando os dados encontrados na pesquisa Rover et al. (2009). Já a categoria informações financeiras ambientais teve seus indicadores mais evidenciados pelo segmento de Extração e Tratamento de Minerais. Na análise global, a categoria outras informações ambientais apresentou-se como a que mais teve seus indicadores evidenciados pelas corporações, e a categoria informações financeiras ambientais a que menos evidenciou, ratificando as pesquisas de Rover et al. (2009) e Macêdo et al. (2013).

A análise individual dos indicadores demonstrou que políticas ambientais é o mais evidenciado pelas corporações em suas páginas eletrônicas de Internet, corroborando pesquisas anteriores (Yusoff et al., 2006; Murcia et al., 2009; Rover et al., 2009; Macêdo et al., 2013; Souza et al., 2013; Correa et al., 2015), seguido dos indicadores controle de poluição e preservação/conservação. Esses resultados sugerem que as corporações acreditam em sua responsabilidade com o meio ambiente e estão comprometidas com as questões ambientais, mas também reforça a teoria que as corporações tendem a evidenciar mais informações positivas em prol de sua imagem e da legitimação.

No entanto, os resultados também demonstram que ainda é pouca a transparência de informações que interessam ao público de interesse das corporações. Frisa-se que os stakeholders estão cada vez mais interessados nas informações ambientais corporativas e, assim, a evidenciação ambiental desempenha um importante papel para suprir as necessidades deles e garantir a sobrevivência da corporação (Patten, 1992; Deegan, 2002; Yusoff et al., 2006; Cho \& Patten, 2007; Ortas et al., 2014). A falta de evidenciação ou o baixo nível dela afeta os stakeholders influenciando em suas tomadas de decisão e também prejudica a corporação, haja vista elas não fornecerem informações suficientes para seus stakeholders reconhecê-las como legítimas (Lindblom, 1994; Patten, 1992; Suchman, 1995; Deegan, 2002; O’Donovan, 2002; Phillips, 2003).

Todavia, a ausência das informações nas páginas eletrônicas de Internet das corporações, apesar de sinalizar que estas não as divulgam, não significa a não evidenciação, pois outros meios de comunicação podem estar sendo utilizados. Além disso, percebe-se que a quantidade de informações ambientais, com acesso facilitado para os stakeholders, ainda é baixa, principalmente, quando se trata de corporações ambientalmente sensíveis por serem mais vulneráveis a pressões públicas (Patten, 1992; Deegan, 2002; Yusoff et al., 2006; Cho \& Patten, 2007; Ortas et al., 2014). 
Por fim, os resultados encontrados demonstraram a variação na evidenciação dos indicadores pelas corporações. Notou-se que os maiores índices de evidenciação apresentaram menores desvios padrão, enquanto que nos baixos índices o desvio padrão é maior. Essa variação nos indicadores evidenciados também foi confirmada por meio do teste das médias Anova, no qual o resultado não rejeitou a hipótese 1 de que as médias da evidenciação dos indicadores ambientais não são iguais. Isso mostra a falta de padronização na divulgação de informações ambientais e como existem diferenças nas estratégias de evidenciação adotadas pelas corporações, para se alcançar a legitimação. Dessa forma, esse estudo pode contribuir com os órgãos reguladores, no sentido de padronizar a evidenciação de informações ambientais, oferecendo uma relação de indicadores básicos.

Esta pesquisa teve limitações, pois se fez uso apenas de informações disponíveis no meio eletrônico. Não se verificou a veracidade das informações divulgadas e não foi analisado o seu grau de importância. Assim, sugere-se que novos estudos sejam realizados, considerando o que não foi abrangido nesta pesquisa, a fim de contribuir para melhoria das informações ambientais que estão sendo divulgadas para os stakeholders, objetivando a qualidade dessa evidenciação.

\section{Referências}

Bardin, L. (1977). Análise de conteúdo. São Paulo: Edições 70.

Bebbingont, J., Larrinaga, C. \& Moneva, J. M. (2008). Corporate social reporting and reputation risk management. Accounting, Auditing \& Accountability Journal, 21(3), 337-361.

Beuren, I. M. \& Söthe, A. (2009). A teoria da legitimidade e o custo político nas evidenciações contábeis dos governos estaduais da região sudeste do Brasil. Revista de Contabilidade e Organizações, 3(5), 98-120.

Beuren, I. M., Hein, N. \& Boff, M. L. (2011). Estratégias de legitimidade organizacional de Lindblom versus geração familiar gestora de empresas familiares. Revista Iberoamericana de Contabilidad de Gestión, 9(17), 56-72.

Burgwal, D. V. de \& Vieira, R. J. O. (2014). Determinantes da divulgação ambiental em companhias abertas holandesas. Revista Contabilidade \& Finanças, 25(64), 60-78. 
Cho, C. H., Freedman, M. \& Patten, D. M. (2012). Corporate disclosure of environmental capital expenditures: A test of alternative theories. Accounting, Auditing \& Accountability Journal, 25(3), 486-507.

Cho, C. H., \& Patten, D. M. (2007). The role of environmental disclosures as tools of legitimacy: A research note. Accounting, Organizations and Society, 32(7), 639-647.

Clarkson, P. M., Xiaohua, F., Li, Y. \& Richardson, G. (2013). The relevance of environmental disclosures: Are such disclosures incrementally informative? Journal of Accounting and Public Policy, 32(5), 410-431.

Conselho Federal de Contabilidade. (2004). NBC - T 15 - Informações de natureza social e ambiental. Recuperado em 28 de março, 2016, de http://www.portaldecontabilidade.com.br/nbc/t15.htm:

Cormier, D. \& Magnan, M. (2015). The economic relevance of environmental disclosure and its impact on corporate legitimacy: An empirical investigation. Business Strategy and the Environment, 24(6), 431-450.

Correa, J. C., Gonçalves, M. N. \& Moraes, R. de O. (2015). Disclosure ambiental das companhias do setor petróleo, gás e biocombustíveis listadas na BM\&FBOVESPA: Uma análise à luz da teoria da legitimidade. Revista de Gestão Ambiental e Sustentabilidade, 4(3), 139-154.

Dantas, J. A., Zendersky, H. C., Santos, S. C. dos \& Niyama, J. K. (2008). A dualidade entre os benefícios do disclosure e a relutância das organizações em aumentar o grau de evidenciação. Revista Economia \& Gestão, 5(11), 56-76.

Deegan, C. (2002). Introduction: the legitimizing effect of social and environmental disclosures-a theoretical foundation. Accounting, Auditing \& Accountability Journal, $15(3), 282-311$.

Deegan, C. \& Rankin, M. (1996). Do Australian companies report environmental news objectively? An analysis of environmental disclosures by firms prosecuted successfully by the environmental protection authority. Accounting, Auditing \& Accountability Journal, 15(3), 282-311.

Dye, R. A. (1986). Proprietary and nonproprietary disclosures. The Journal of Business, 59(2), 331-366. 
Elijido-Ten, E.; Kloot, L. \& Clarson, P. (2010). Extending the application of stakeholder influence strategies to environmental disclosures: An exploratory study from a developing country. Accounting, Auditing \& Accountability Journal, 23(8), 1032-1059.

Ezhilarasi, G. \& Kabra, K. C. (2017). Factors influencing environmental disclosures: Evidence from India. Journal of Accounting Research \& Audit Practices, 16(1), 7-24.

Fifka, M. S. (2013). Corporate Responsibility Reporting and its Determinants in Comparative Perspective - a Review of the Empirical Literature and a Meta-analysis. Business Strategy and the Environment, 9(2), 50-67.

Freeman, E. R. (2002). Stakeholder theory of the modern corporation. Cambridge University Press.

Gallopin, G. C. (1996). Environmental and sustainability indicators and the concept of situational indicators. A system approach. Environmental Modelling \& Assessment, 1, 101-117.

Global Reporting Iniciative (2015). Diretrizes para relatório de sustentabilidade. Recuperado em 28 de março, 2016, de https://www.globalreporting.org/resourcelibrary/GRI-G3Brazilian-Portuguese-Reporting-Guidelines.pdf.

Hammond, A. et al. (1995). Environmental indicators: a systematic approach to measuring and reporting on environmental policy performance in the context of sustainable development. Washington, D.C.: World Resources Institute.

Hardi, P. \& Barg, S. (1997). Measuring sustainable development: review of current practice. Winnipeg: IISD.

Lei $n$. 10.165, de 27 de dezembro de 2000 (2000). Altera a Lei $\mathrm{n}^{\mathrm{o}}$ 6.938, de 31 de agosto de 1981 que dispõe sobre a Política Nacional do Meio Ambiente, seus fins e mecanismos de formulação e aplicação, e dá outras providências. Brasília, 2000. Recuperado em 14 maio, 2016, de http://www.planalto.gov.br/ccivil_03/leis/L10165.htm.

Lindblom, C. K. (1994). The implications of organizational legitimacy for corporate social performance and disclosure. In: Critical Perspectives on Accounting Conference, New York. 
Macagnan, C. B. \& Silva, V. (2012). Categorias de informações evidenciadas nos relatórios anuais. Redes, 17(2), 154-174.

Macêdo, F. F. R. R., Moura, G. D. de Dagostini, L. \& Hein, N. (2013). Evidenciação ambiental voluntária e as práticas de governança corporativa de empresas listadas na BM\&FBOVESPA. Contabilidade, Gestão e Governança, 16(1), 3-18.

Marquezan, L. H. F., Seibert, R. M., Bartz, D., Barbosa, M. A. G. \& Alves, T. W. (2015). Análise dos determinantes do disclosure verde em relatórios anuais de empresas listadas na BM\&FBOVESPA. Contabilidade, Gestão e Governança, 18(1), 127-150.

Martins, G. A. \& Domingues, O. (2011). Estatística geral e aplicada (4a ed.). São Paulo: Atlas.

Michelon, G. (2011). Sustainability disclosure and reputation: A comparative study. Corporate Repution Review, 14(2), 79-96.

Mir, M. Z. \& Rahaman, A. S. (2011) In pursuit of environmental excellence: A stakeholder analysis of the environmental management strategies and performance of an Australian energy company. Accounting, Auditing \& Accountability Journal, 24(7), 848-878.

Morhardt, J. E. (2010). Corporate social responsibility and sustainability reporting on the internet. Business Strategy and the Environment, 19(7), 436-452.

Murcia, F. D. R., Fávero, L. P. L., Rover, S., Lima, G. A. S. F. \& Lima, I. (2008). “Disclosure Verde" nas demonstrações contábeis: características da informação ambiental e possíveis explicações para a divulgação voluntária. Contabilidade, Gestão $e$ Governança, 11(1-2), 260-278.

Murcia, F. D. R., \& Santos, A. (2009). Fatores determinantes do nível de disclosure voluntário das companhias abertas no Brasil. Revista de Educação e Pesquisa em Contabilidade (REPeC), 3(2), 72-95.

Nor, N. M., Bahari, N. A. S., Adnan, N. A., Kamal, S. M. Q., \& Ali, I. M. (2016). The effect of environmental disclosure on financial performance in Malaysia. Procedia Economics and Finance, 35, 117-126.

Nossa, V. (2002). Disclosure ambiental: uma análise do conteúdo dos relatórios ambientais de empresas do setor de papel e celulose em nível internacional. Tese de Doutorado, Universidade de São Paulo, SP, Brasil. 
O'Donovan, G. (2002). Environmental disclosures in the annual report: Extending the applicability and predictive power of legitimacy theory. Accounting, Auditing \& Accountability Journal, 15(3), 344-371.

Ortas, E., Gallego-Alvarez, I. \& Etxeberria, I. A. (2014). Financial factors influencing the quality of corporate social responsibility and environmental management disclosure: A quantile regression approach. Corporate Social Responsibility and Environmental Management, 22(6), 362-380.

Parmar, B. L., Freeman, R. E., Harrison, J. S., Wicks, A. C., Purnell, L. \& Colle, S. (2010). Stakeholder theory: The state of the art. The Academy of Management Annals, 4(1), 403-445.

Patten, D. M. (1992). Intra-industry environmental disclosures in response to the Alaskan oil spill: A note on legitimacy theory. Accounting, Organizations and Society, 17(5), 471475.

Patten, D. M. (2002). The relation between environmental performance and environmental disclosure: a research note. Accounting, Organizations and Society, 27(8), 763-773.

Phillips, R. (2003). Stakeholder legitimacy. Business Ethics Quarterly, 13, 25-41.

Rempel, R. S., Andison, D. W. \& Hannon, S. J. (2004). Guiding principles for developing an indicator and monitoring framework. The Forestry Chronicle, 80(1), 82-90.

Rover, S.; Borba, J. A. \& Murcia, F. D. R. (2009). Características do disclosure ambiental de empresas brasileiras potencialmente poluidoras: análise das demonstrações financeiras e dos relatórios de sustentabilidade do período de 2005 a 2007. Contextus - Revista Contemporânea de Economia e Gestão, 7(1), 23-36.

Rover, S.; Murcia, F. D., \& Murcia, F. C. S. (Fall/2015). The determinants of social and environmental disclosure practices: The Brazilian case. Environmental Quality Management, 25(1), 05-24.

Rover, S., Tomazzia, E. C., Murcia, F. D. R. \& Borba, J. A. (2012). Explicações para a divulgação voluntária ambiental no Brasil utilizando a análise de regressão em painel. Revista de Administração, 47(2), 217-230. 
Shocker, A. D. \& Sethi, S. P. (1973). An approach to incorporating societal preferences in developing corporate action strategies. California Management Review, XV (4), 97-105.

Souza, J. L., Sousa, A. L. S., Parente, P. H. N., Ferreira, F. E. F. R., \& Souza Filho, F. A. (2013). Disclosure ambiental de empresas listadas na BM\&FBOVESPA: Avaliação de 19 itens do modelo ISAR/UNCTAD em 40 empresas. Anais do Encontro Nacional de Engenharia de Produção, Salvador, BA, Brasil, 33.

Suchman, M. C. (1995). Managing legitimacy: Strategic and institutional approaches. Academy of Management Review, 20(3), 571-610.

Van Bellen, H. M. (2004). Indicadores de sustentabilidade: um levantamento dos principais sistemas de avaliação. Cadernos Ebape.BR, II(1), 2004.

Vergara, S. C. (2010). Métodos de pesquisa em administração (4a ed.). São Paulo: Atlas.

Verrecchia, R. E. (1983). Discretionary disclosure. Journal of accounting and economics, 5, 179-194.

Verrecchia, R. E. (2001). Essays on disclosure. Journal of accounting and economics, 32(1), 97-180.

Wagenhofer, A. (1990). Voluntary disclosure with a strategic opponent. Journal of Accounting \& Economics, 12, 341-363.

Watts, R. L. \& Zimmerman, J. L. (1978). Towards a positive of the determination of accounting standards. The Accounting Review, 53(1), 112-134.

Yusoff, H., Lehman, G. \& Nasir, N. M. (2006). Environmental engagements through the lens of disclosure practices: a Malaysian story. Asian Review of Accounting, 14(1/2), 122148. 